\title{
WIDEBAND MULTIPATH PROPAGATION FOR HELICOPTER-TO-GROUND TELEMETRY LINKS
}

\author{
Michael Rice and Michael Jensen \\ Brigham Young University \\ Provo, Utah, USA
}

KEYWORDS

multipath propagation, power delay profile, diversity gain, L-band

\begin{abstract}
This paper reports the analysis of L-band channel sounding experiments conducted along the flight line at Cairns Army Airfield, Ft. Rucker, Alabama. Propagation data from multiple antennas on a helicopter to multiple receiving antennas on the ground are used to compute power delay profiles. Analysis of the results reveals delay spreads of the multipath channels between $200 \mathrm{~ns}$ and $400 \mathrm{~ns}$, with the longer delay spreads resulting when using a receive antenna with lower gain and higher sidelobe levels. The data also shows that on average, diversity signaling from three aircraft-mounted antennas can lead to gains in signal-to-noise ratio of approximately $13 \mathrm{~dB}$, with the gain dependent on the multipath characteristics observed by the ground antenna.
\end{abstract}

\section{INTRODUCTION}

Understanding multipath propagation allows telemetry engineers to identify and evaluate the performance of multipath mitigation techniques. Because multipath propagation is the dominant channel impairment for low-elevation angle air-to-ground links, effective multipath mitigation has the prospect of improving the overall link availability. Whether or not a multipath mitigation technique can be identified as "effective" depends on the accuracy of the multipath propagation data used to test it. To this end, multipath propagation experiments are conducted to collect data for the scenarios and frequency bands of interest.

In its current form, the typical aeronautical telemetry system comprises an airborne transmitter and a ground station equipped with a large tracking antenna. The relatively narrow beamwidth of 


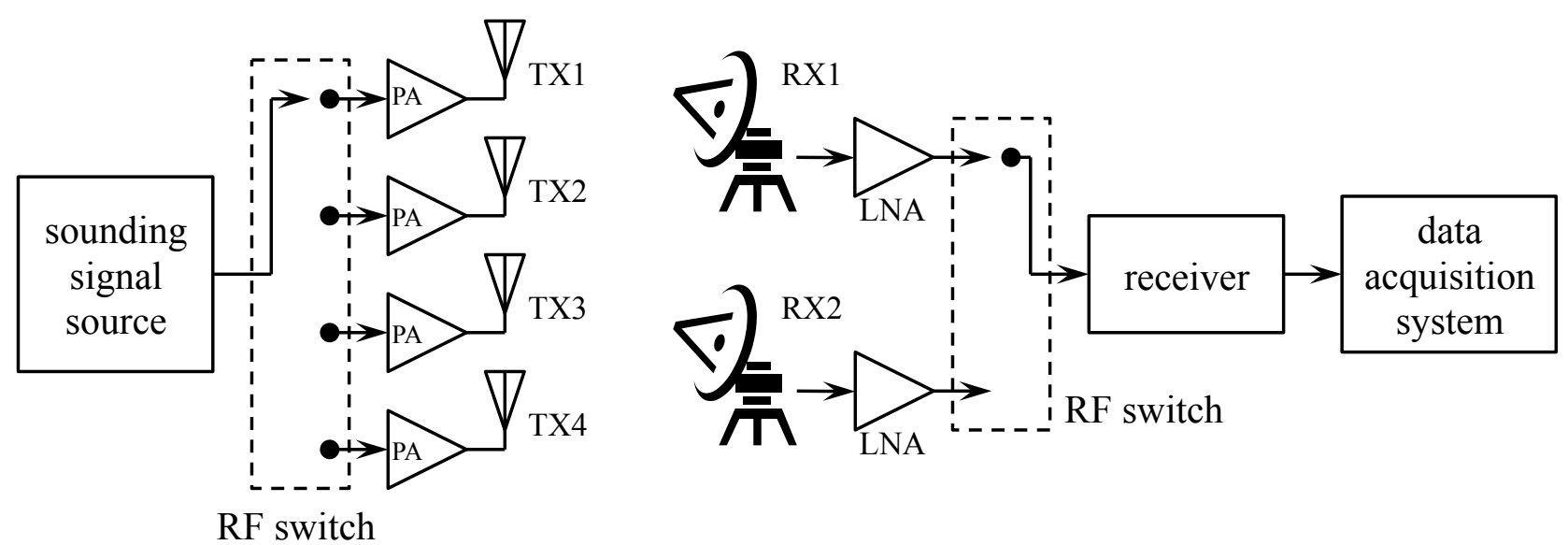

Figure 1: A block diagram of the system used for the channel sounding experiments.

the ground-based receive antenna tends to attenuate off-boresite reflections in the propagation path for "up and away" flight profiles. However, low-elevation-angle and flight-line scenarios present serious challenges. Previous work in this area include experiments conducted at the Air Force Flight Test Center, Edwards Air Force Base in L- and S-bands [1] and at Pt. Mugu Naval Air Station over the Pacific Ocean in X-band [2]. These experiments provided useful data for lowelevation-angle propagation in the "up and away" scenario at test ranges in the western United States. One shortcoming of these experiments, however, is the absence of flight-line propagation data.

Channel sounding experiments conducted on the flight-line at Cairns Army Airfield, Ft. Rucker, Alabama in June 2010 are the first channel sounding experiments dedicated to flight-line propagation. This data will improve our understanding of multipath propagation in one of the most difficult scenarios test engineers usually encounter and help in identifying mitigation techniques that make reliable communication possible.

Another unique feature of these experiments is the use of multiple transmit and receive antennas. Thus, these experiments provide data that is relevant for the analysis of both equalization and spatial diversity techniques. We show that in this environment, delay spreads of the multipath channels vary from about $200 \mathrm{~ns}$ to $400 \mathrm{~ns}$, with the variability strongly dependent on the transmit antenna placement and the receive antenna radiation characteristics. We also show that on average, diversity signaling from multiple aircraft-mounted antennas can lead to gains in signal-to-noise ratio (SNR) of approximately $13 \mathrm{~dB}$, with the gain notably dependent on the multipath characteristics observed by the ground antenna.

\section{EXPERIMENTAL CONFIGURATION}

A block diagram of the system is illustrated in Figure 1. The channel sounding signal comprised 501 unmodulated tones with a 100-kHz spacing. The occupied bandwidth was $50 \mathrm{MHz}$. The RF channel sounding signal, centered at $1800 \mathrm{MHz}$ (upper L-band) was applied to a Herley F9140W 


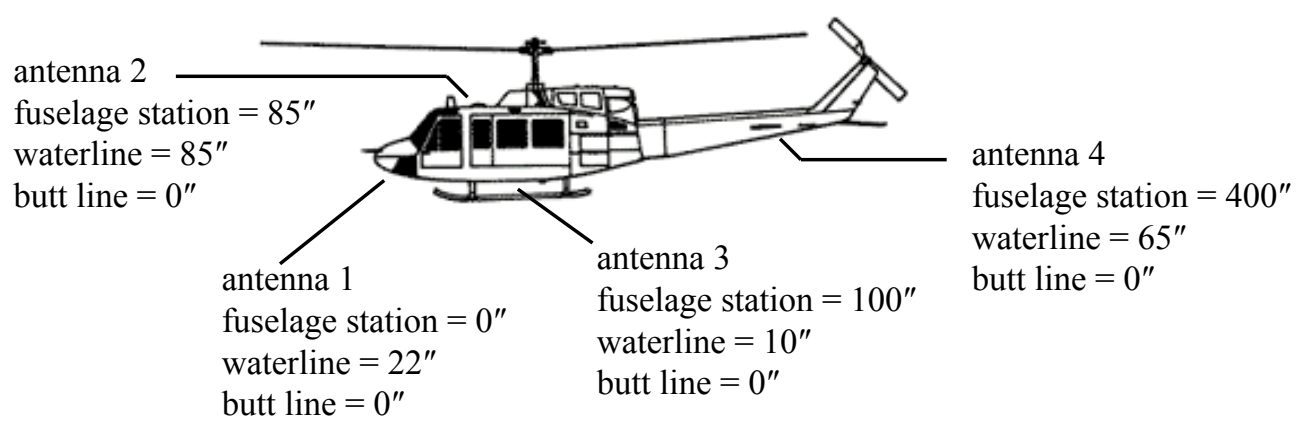

Figure 2: A diagram of the UH-1H airborne platform showing the transmit antenna locations.

Table 1: Description of the receive antennas.

\begin{tabular}{|c|c|l|}
\hline Antenna & diameter & lomments: \\
\hline RX1 & $6 \mathrm{ft.}$ & $\begin{array}{l}\text { elevation }=60 \mathrm{ft} . \text { AGL, tracking per- } \\
\text { formed by conical scan }\end{array}$ \\
\hline RX2 & $4 \mathrm{ft}$. & $\begin{array}{l}\text { elevation }=60 \mathrm{ft} . \text { AGL, tracking per- } \\
\text { formed by steering using GPS data } \\
\text { downlinked from the UH-1H }\end{array}$ \\
\hline
\end{tabular}

RF switch as shown. The dwell time for each switch position was $50 \mu \mathrm{s}$ ( 5 periods of the channel sounding signal). The four switch outputs were connected to separate $10-\mathrm{W}$ Remec RF power amplifiers each cabled to a transmit antenna. The transmit antennas were small blade antennas (UB Corp. AO4459) mounted on the fuselage of a UH-1H helicopter as illustrated in Figure 2.

The ground station (receiver) used two dish antennas with tracking capability, the details of which are summarized in Table 1. The RF outputs from each receive antenna were routed to a single receiver through a second switch, switched in synchronism with the transmit switch through disciplined rubidium oscillators. The dwell time for each receive antenna was $250 \mu \mathrm{s}$, which accommodated one $50 \mu \mathrm{s}$ slot for each of the four transmit antennas and one $50 \mu \mathrm{s}$ blank period used for synchronization during data post-processing. The RF switch output was applied to a Cobham M/A-COM SMR-5550i microwave receiver, and the resulting intermediate frequency (70 MHz) signal was sampled at 200 Msamples/s by a Wideband Systems (DRS2200-144GB-2CHA1) data acquisition system and stored. GPS data were also recorded on the aircraft and incorporated into the post-processing.

The flight path together with the receive antenna locations are illustrated in Figure 3. The UH$1 \mathrm{H}$ flew along the flight path called the "ramp" at an altitude of $15-20$ feet above ground level (AGL). The receive antennas, shown near the bottom of Figure 3, were elevated to 60 feet AGL using dedicated towers. This arrangement is used at Cairns Army Airfield to allow clearance over the buildings and hangars positioned between the runways (not shown) and the main telemetry receiving center. The "ramp" area presents a particularly troublesome area because typical helicopter altitudes are insufficient to produce line-of-sight (LOS) propagation. The hangars and buildings not only shadow the transmitted signal, but also provide ample opportunity for strong multipath 
propagation.

Samples of the band limited version of the channel impulse response were generated by examining the received signal over one sounding signal period of $10 \mu \mathrm{s}$. Denote by $s(t)$ the transmitted sounding signal by $r(t)$ the received signal. The relationship between the two is

$$
r(t)=s(t) * h(t)+w(t)
$$

where $h(t)$ is the unknown channel, $*$ is the convolution operation, and $w(t)$ is the additive thermal noise. The goal is to estimate samples of $h(t)$ from samples of one period of $r(t)$ and $s(t)$. Let $T$ be the sample time where $1 / T=200 \times 10^{6}$ samples/s and let $r(n T)$ and $s(n T)$ be the $n$-th sample of $r(t)$ and $s(t)$, respectively. Because there are $N=2000$ samples in a period, we have $0 \leq n<N$. The required deconvolution operation is performed in the frequency domain. Let $R\left(e^{j 2 \pi k / N}\right)$ and $S\left(e^{j 2 \pi k / N}\right)$ be the $k$-th sample of the length- $N$ DFT of $r(t)$ and $s(t)$, respectively, for $0 \leq k<N$. Then the $k$-th sample of DFT of the channel is

$$
\hat{H}\left(e^{j 2 \pi k / N}\right)=\frac{R\left(e^{j 2 \pi k / N}\right)}{S\left(e^{j 2 \pi k / N}\right)} .
$$

Notes:

1. The relationship $R\left(e^{j 2 \pi k / N}\right)=H\left(e^{j 2 \pi k / N}\right) S\left(e^{j 2 \pi k / N}\right)$ defines the frequency-domain relationship for the periodic extensions of $r(n T), h(n T)$, and $s(n T)$. As such, the corresponding time domain relationship $r(n T)=h(n T) * s(n T)$ is thought of as a circular convolution [3]. In general, the circular convolution and linear convolution are not equivalent. However, because the transmitted signal is periodic (and one period of the transmitted and received signals are used), the two convolutions are equivalent and (2) gives the desired result.

2. Because the samples $s(n T)$ define a real-valued bandpass signal, the corresponding DFT The division defined in (2) is only performed for the indexes corresponding to the region of support for $S\left(e^{j 2 \pi k / N}\right)$. Let this region of support be defined by the indexes $K_{1} \leq k \leq K_{2}$. Given the bandwidth, period, and sample rate, we have $K_{2}-K_{1}=500$. Consequently, there are 501 points in the region of support so that $\hat{H}\left(e^{j 2 \pi k / N}\right)$ is defined by 501 samples.

3. The discrete-time impulse response $\hat{h}(n T)$ is the inverse DFT of $\hat{H}\left(e^{j 2 \pi k / N}\right)$ for $K_{1} \leq k \leq$ $K_{2}$. The sample spacing for $\hat{h}(n T)$ is $5 \mathrm{~ns}$ (as determined by the sample rate).

\section{EXPERIMENTAL RESULTS}

The sampled channel frequency responses measured along the ramp for each pair of transmit and receive antennas ${ }^{1}$ are reduced to a sample rate of 200 samples/second, providing a sampling in spatial displacement of the aircraft of approximately 10 samples per wavelength at the center frequency of the transmission. Because of uncertainties regarding the responses of cables and electronics for each transmit and receive antenna, it is necessary to perform calibration on the observed

\footnotetext{
${ }^{1}$ The UH-1H GPS antenna was located on the top near TX 2. Transmit antenna 2 was turned off for the L-band channel sounding experiments because it interfered with reception of the GPS signal.
} 


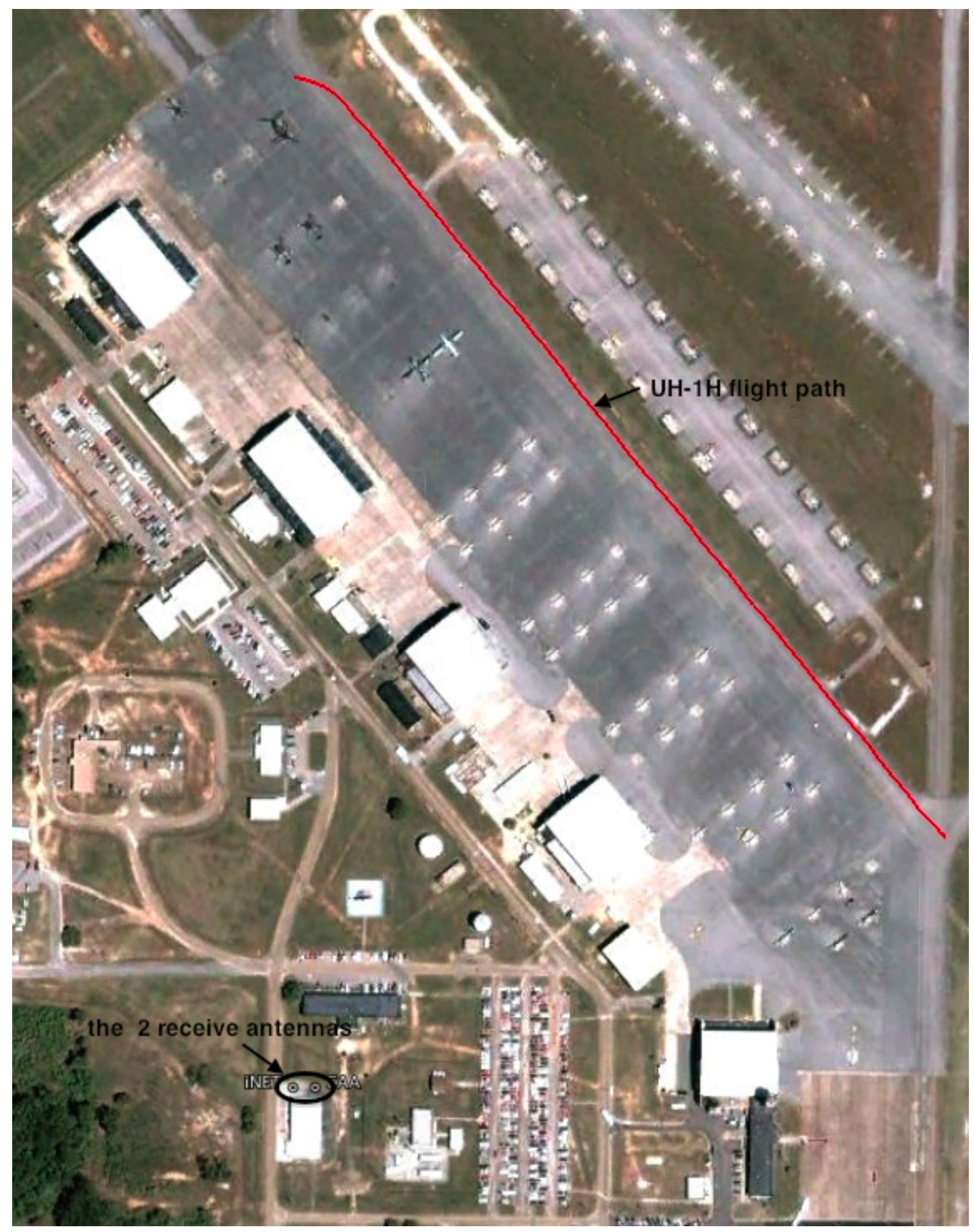

Figure 3: The flight path at Cairns Army Airfield, Ft. Rucker, AL. The UH-1H flight path is the "ramp" area shown. The two receive antennas are located on towers situated at the bottom of the figure. 
channels before using them to analyze the channel behavior. This calibration involves power normalization and temporal alignment of the channel impulse responses for each antenna pair. In the following, $\hat{H}_{i j}^{(q)}\left(e^{2 \pi k / N}\right)$ represents the frequency response from the $j$ th transmit antenna to the $i$ th receive antenna at the $q$ th sample time. The corresponding discrete-time channel impulse response is designated as $\hat{h}_{i j}^{(q)}(n T)$, where we emphasize that $n T$ represents the discretized delay variable.

The power normalization must take into consideration the fact that the receive system uses automatic gain control (AGC) at the intermediate frequency, with the goal of ensuring that each received waveform has the same total received power. As the switches connect different antennas with varying gains, however, the AGC cannot always respond quickly enough to guarantee achievement of this goal. To remove the impacts of the notably different gains associated with the two receive antennas, we scale the responses observed on RX1 and RX2 by different values. Therefore, we compute the total power for the $q$ th channel transfer function from TX3 to RX1 and from TX3 to RX2, denoted respectively as $P_{\mathrm{T}, 1}^{(q)}$ and $P_{\mathrm{T}, 2}^{(q)}$. We then normalize the channel transfer functions as $\hat{H}_{i j}^{(q)} \leftarrow \hat{H}_{i j}^{(q)} / \sqrt{P_{\mathrm{T}, i}^{(q)}}$ (functional notation dropped). This preserves the relative scaling between responses for different transmit antennas, since these gains are dictated by antenna placement and not by differences in antenna gain.

With the gains properly normalized, we compute the impulse responses using an inverse DFT. Differences in delays observed in the responses from different transmit antennas are due to different cable lengths to each antenna as well as the different positions of the antennas on the airframe, and these differences are small compared to the delay spreads observed in the multipath channels. In contrast, differences in delays observed in the responses from different receive antennas are significant due to different electronic subsystems and the long cables used to connect these subsystems. Therefore, we align the responses observed on RX1 and RX2 using different temporal shifts. Specifically, for the two channels between TX3 and RX1 and between TX3 and RX2, we define the beginning of the channel impulse response as the first sample at which its magnitude reaches $20 \%$ of its peak value, denoted respectively as $n_{1}^{(q)}$ and $n_{2}^{(q)}$. Using this definition reduces sensitivity to noise in the waveform and has been found to be robust for accurately detecting the beginning of each response. We then designate sample $n_{i}^{(q)}$ as zero delay for all three impulse responses observed on $\mathrm{RX} i$.

\section{A. Power Delay Profiles}

Given this waveform calibration, we are prepared to examine the multipath delay structure. Specifically, we compute the power delay profile (PDP) of the impulse responses using [4]

$$
\operatorname{PDP}_{i j}(n T)=\frac{1}{Q_{\mathrm{m}}} \sum_{q=1}^{Q_{\mathrm{m}}}\left|\hat{h}_{i j}^{(q)}(n T)\right|^{2}
$$

where $Q_{\mathrm{m}}$ is the total number of temporal channel samples over the measurement. This PDP provides an average measure of the power as a function of delay in the multipath channel, and it both gives an indication of the type of fading that might be observed in typical channels and helps 

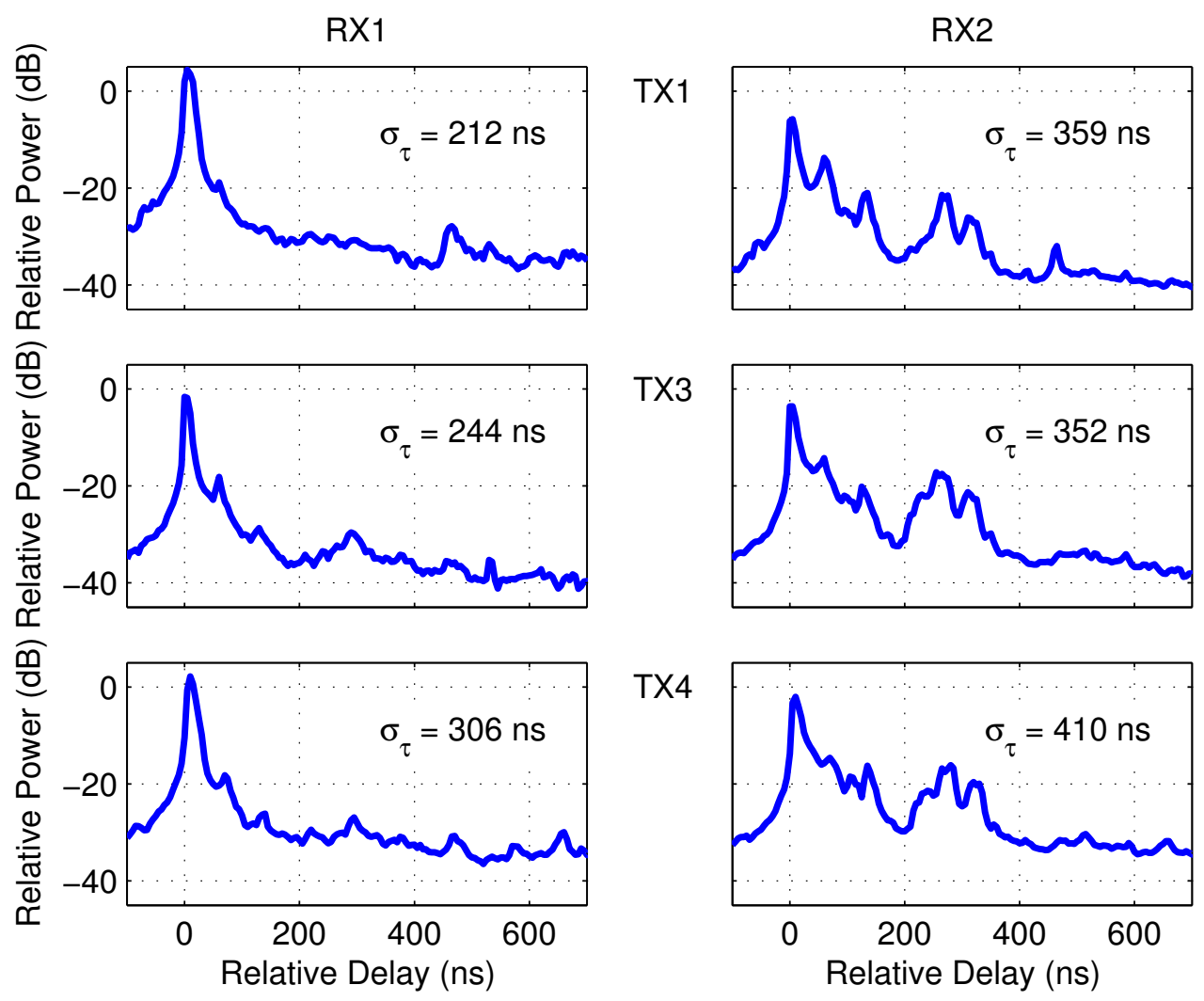

Figure 4: Power delay profiles observed for each pair of transmit and receive antennas while the helicopter moves along the ramp along with the calculated average delay spreads for each PDP.

to define the complexity of the equalizer that can adequately compensate for the range of multipath delay signatures observed.

Figure 4 plots the PDP waveforms for each transmit-receive antenna pair. Several observations are immediately obvious from these figures. First of all, when compared with the responses observed on $\mathrm{RX} 2$, the responses observed on $\mathrm{RX} 1$ clearly demonstrate less power arriving in multipaths with long delays. We believe this is due to the fact that long delays are likely to arrive at angles that are notably different from the LOS angle, and since RX1 is more directive than RX2, it does not observe as many of these multipath components. Second, we notice that the components with long delays observed on RX2 appear in a second cluster of power (centered at approximately $300 \mathrm{~ns}$ in Fig. 4). The reason for this clustering is not entirely clear. However, one possible explanation is that the radiation pattern of RX2 has sidelobes that are higher than those for the pattern of RX1. Since the components with longer delays likely arrive at wide angles, these may be observed on the sidelobes of RX2. Under such circumstances, multipath components with delays around $200 \mathrm{~ns}$ might arrive at angles near the first null in the pattern of RX2, which would result in the bi-modal behavior observed.

These PDP plots provide a visual indication of the extent of delay experienced for the observed channels. However, we can further quantify this delay extent using the average delay spread for 
each channel. The average delay spread $\sigma_{\tau, i j}$ for each PDP can be computed using [4]

$$
\sigma_{\tau, i j}^{2}=\frac{1}{P_{i j}} \sum_{n}(n T)^{2} \operatorname{PDP}_{i j}(n T)-\bar{\tau}^{2}
$$

where

$$
\begin{aligned}
P_{i j} & =\sum_{n} \operatorname{PDP}_{i j}(n T) \\
\bar{\tau} & =\frac{1}{P_{i j}} \sum_{n}(n T) \operatorname{PDP}_{i j}(n T) .
\end{aligned}
$$

The delay spread values are included in the PDP plots of Fig. 4. As can be observed, the responses on RX2 have notably larger delay spread values, consistent with the late arrivals of multipath components discussed previously. We also see that for both receive antennas, the delay spread for TX4 is notably larger than that for the other antennas. There are at least two factors that likely contribute to this observation. First, TX4 is on the tail of the helicopter, and the time reference is taken with respect to TX3 on the helicopter belly. As the aircraft attitude changes, the difference in the range to the receive antennas between the belly and tail antennas (TX3 and TX4) can vary by as much as 25 feet in either direction, resulting in about $50 \mathrm{~ns}$ of arrival variability for TX4 relative to that for TX3. Second, the position of TX4 makes it more likely for signals to bounce off the lower portion of the main helicopter fuselage and propagate to the receiver, resulting in multipath components with additional delay.

In interpreting these results, it is also helpful to understand that the two receive antennas use different tracking mechanisms. RX1 uses a conical scan to maximize the received signal. Therefore, if the LOS path is obscured, RX1 likely will track on a multipath component, and with the higher gain and narrower beamwidth will be less likely to see other multipath components. In contrast, RX2 tracks by pointing to the GPS coordinates of the aircraft (communicated through a dedicated telemetry link). This means it will point at the aircraft regardless of the quality of the LOS signal, possibly resulting in reduced overall power but richer multipath observations.

\section{B. Diversity Gain}

The theoretically achievable diversity gain for the channels was computed using the procedure outlined in [5]. This is a purely theoretical result whose intent is to demonstrate the potential performance gain using diversity. Figure 5 shows the complementary cumulative distribution function (CCDF) of the achieved diversity gain for each receive antenna assuming the gain for each frequency and spatial sample is treated as a realization of the random variable. Given that RX2 observes richer multipath than $\mathrm{RX} 1$, it is not surprising that using diversity on the aircraft generally achieves higher diversity gain for RX2. In considering this result, however, we emphasize that diversity gain represents the improvement in achieved SNR relative to that of a single-antenna link using the reference channel. If, for example, the reference link has very poor SNR, the gain obtained using diversity can be significant. However, higher diversity gain for RX2 does not necessarily mean that the overall SNR observed on RX2 is higher than that observed on RX1, but simply that diversity offers increased relative improvement. 


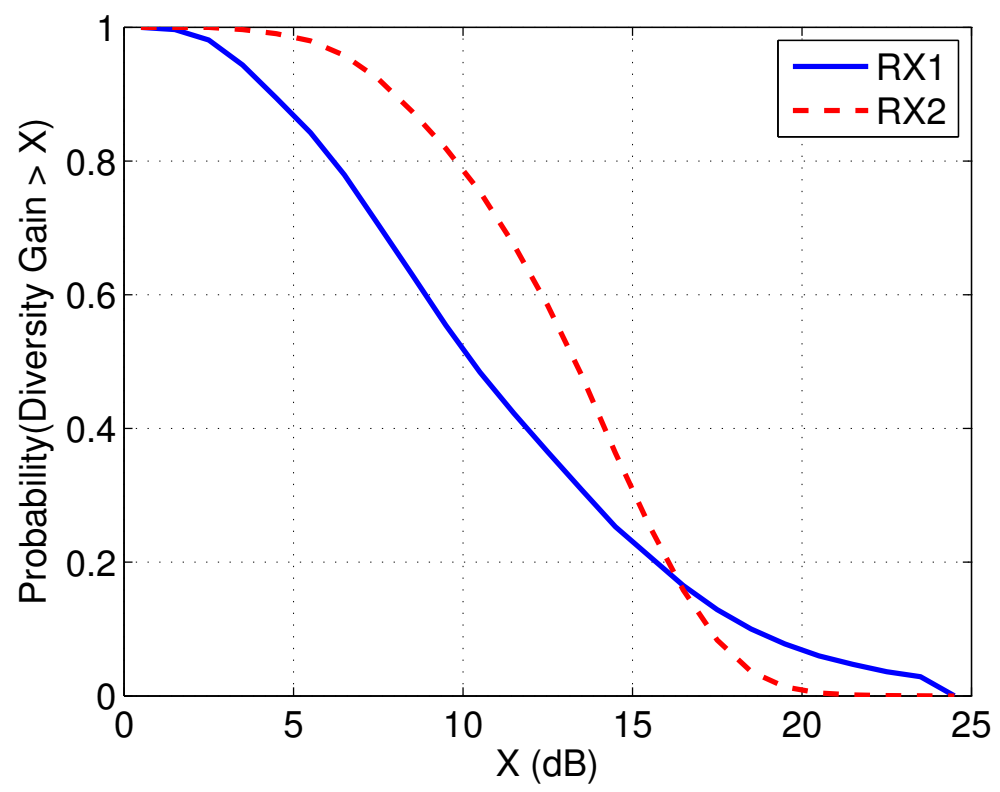

Figure 5: CCDF of the diversity gain achievable at each receive antenna over all frequencies and spatial channel samples along the ramp.

It is also interesting that RX1 is more likely to experience very high diversity gain than RX2. Apparently, there are cases where the reference link to RX1 are very poor (such as when the LOS is highly obscured), and the wide spatial separation of the aircraft antennas leads to significant diversity gains. Because RX2 benefits from additional multipath, in these cases the reference response may be stronger, somewhat reducing the effectiveness of diversity signaling. It is noteworthy that this high gain occurs at low probability, which is intuitive.

\section{ACKNOWLEDGEMENTS}

The authors acknowledge the hard work and contributions of Mr. Glen Wolf (Delphi Research, Inc., Air Force Flight Test Center) who put the system together; Mr. Michael Mando (U.S. Army Aviation Technical Test Center, Cairns Army Airfield) who made the local arrangements; Mr. Michael Fox (AFTD, Wyle Labs/WESTAR) and Nick Walters (RTC, US Army) who piloted the helicopter.

This work was supported by the Test Resource Management Center (TRMC) Test and Evaluation Science and Technology (T\&E/S\&T) Program through a grant to BYU from the US Army Program Executive Office for Simulation, Training, and Instrumentation (PEO STRI) under contract W900KK-09-C-0016. Any opinions, findings and conclusions or recommendations expressed in this material are those of the author and do not necessarily reflect the views of the TRMC and T\&E/S\&T Program and/or PEO STRI. The Executing Agent and Program Manager work out of the AFFTC. 


\section{REFERENCES}

[1] M. Rice, A. Davis, and C. Bettwieser. A wideband channel model for aeronautical telemetry. IEEE Transactions on Aerospace and Electronic Systems, 40(1):57-69, January 2004.

[2] Q. Lei and M. Rice. Multipath channel model for over-water aeronautical telemetry. IEEE Transactions on Aerospace and Electronic Systems, 45(2):735 - 742, April 2009.

[3] A. Oppenheim and A. Willsky. Signals and Systems. Prentice Hall, 1996.

[4] R. Vaughan and J. B. Andersen. Channels, Propagation, and Antennas for Mobile Communications. The Institution of Electrical Engineers, London, 2003.

[5] M. Rice and M. Jensen. Mulitpath propagation for helicopter-to-ground MIMO links. In Proceedings of the IEEE Military Communications Conference. 